\title{
A Two-Dimensional Type I Superionic Conductor
}

Alexander J. E. Rettie ${ }^{1,2, *}$, Jingxuan Ding, ${ }^{3}$ Xiuquan Zhou, ${ }^{1}$ Michael J. Johnson, ${ }^{2}$ Christos D. Malliakas, ${ }^{4}$ Naresh C. Osti, ${ }^{5}$ Duck Young Chung ${ }^{1}$, Raymond Osborn, ${ }^{1}$ Olivier Delaire, ${ }^{3}$ Stephan Rosenkranz, ${ }^{1, *}$ Mercouri G. Kanatzidis ${ }^{1,4, *}$

1. Materials Science Division, Argonne National Laboratory, Lemont, IL, 60439, USA

2. Electrochemical Innovation Lab, Department of Chemical Engineering, University College London, Bloomsbury, London, WC1E 6BT, UK

3. Departments of Mechanical Engineering, Materials Science and Physics, Duke University, Durham, NC, 27708, USA

4. Department of Chemistry, Northwestern University, Evanston, IL, 60208, USA

5. Neutron Scattering Division, Oak Ridge National Laboratory, Oak Ridge, TN, 37831, USA

*Correspondence to: a.rettie@ucl.ac.uk, srosenkranz@anl.gov,m-kanatzidis@northwestern.edu

\begin{abstract}
Superionic conductors (SICs) possess liquid-like ionic diffusivity in the solid state, finding wide applicability from electrolytes in energy storage to materials for thermoelectric energy conversion. Type I SICs (e.g., AgI, $\mathrm{Ag}_{2} \mathrm{Se}$, etc.) are defined by an abrupt transition to the superionic state and have so far been found exclusively in three-dimensional crystal structures. Here, we reveal a twodimensional type I SIC, $\alpha-\mathrm{KAg}_{3} \mathrm{Se}_{2}$ by scattering techniques and complementary simulations. Quasielastic neutron scattering and ab initio molecular dynamics simulations confirm that the superionic $\mathrm{Ag}^{+}$ions are confined to sub-nanometre sheets, with the simulated local structure validated by experimental X-ray powder pair-distribution-function analysis. Finally, we demonstrate that the phase transition temperature can be controlled by chemical substitution of the alkali metal ions that comprise the immobile charge-balancing layers. Our work thus extends the known classes of SICs and will facilitate the design of new materials with tailored ionic conductivities and phase transitions.
\end{abstract}

\section{Introduction}

Superionic conductors (SICs) are solids which host liquid-like ionic conductivity, i.e., a diffusion coefficient, $D$ of order $\sim 10^{-5} \mathrm{~cm}^{2} \mathrm{~s}^{-1}$, comparable to that of water at room temperature. ${ }^{1}$ In addition to 
attracting long-standing scientific interest, ${ }^{2,3}$ this rapid ionic diffusion naturally lends itself to wide application in energy storage. ${ }^{4,5}$ Recently, SICs have found utility in thermoelectric energy conversion, as atomic disorder and anharmonicity are linked to low lattice thermal conductivity 6,7 and hence, high thermoelectric efficiency. ${ }^{8}$

SICs generally undergo an order-disorder transition to the superionic state, and can be grouped into three classes by the nature of the transition. ${ }^{1,9}$ Type I SICs exhibit an abrupt transition to the superionic state (e.g., $\mathrm{AgI}, \mathrm{Ag}_{2} \mathrm{Se}$, etc.), ${ }^{10}$ which becomes less well-defined for type II (continuous transition, e.g., $\left.\mathrm{PbF}_{2}, \mathrm{AgCrSe}_{2}\right),{ }^{11,12}$ and non-existent in the case of type III SICs (e.g., the $\beta$ aluminas). ${ }^{13}$ In the superionic state, a non-conducting sublattice remains. This rigid network dictates the available conduction pathways and can be linked in one, two or three dimensions. Twodimensional SICs, where long-range superionic diffusion is confined between immobile layers and ionic conduction is strongly anisotropic, are well represented in types II and III, such as $\mathrm{AgCrSe}_{2}{ }^{14}$ and $\mathrm{Ag}: \beta-\mathrm{Al}_{2} \mathrm{O}_{3}{ }^{15}$ respectively. In both of these types, the underlying layered structures are unchanged at the onset of fast ionic conduction and existing additional atomic sites become increasingly populated with increased temperature. Conversely, the type I SICs known to date have exclusively 3D crystal structures (cubic or hexagonal, cf Table 3.2, ref 1 ), ${ }^{1}$ with the archetypical AgI undergoing a first-order superionic phase transition from an ordered, low symmetry phase to a bodycentered cubic (bcc) arrangement of $\mathrm{I}^{-}$ions $(\alpha-\mathrm{AgI})$ with significant $\mathrm{Ag}^{+}$ion disorder at $420 \mathrm{~K}^{16}$ Similarly, orthorhombic $\beta-\mathrm{Ag}_{2} \mathrm{Se}$ transforms to the bcc superionic phase, $\alpha-\mathrm{Ag}_{2} \mathrm{Se}$ at $406 \mathrm{~K} .{ }^{17}$

Previously, we identified a first order, order-disorder phase transition in $2 \mathrm{D} \mathrm{KAg}_{3} \mathrm{Se}_{2}-\mathrm{a}$ dimensionally-reduced derivative of 3D $\mathrm{Ag}_{2} \mathrm{Se}-$ at $\sim 695 \mathrm{~K}$ using in-situ X-ray diffraction and differential scanning calorimetry (Figure S1 in the Supporting Information (SI)). ${ }^{18}$ However, the elevated temperature of the transition prevented measurement of the ionic conductivity because of reactions between $\mathrm{KAg}_{3} \mathrm{Se}_{2}$ and the electrode materials. Here, we use quasi-elastic neutron scattering (QENS) and complementary ab initio molecular dynamics (AIMD) simulations to reveal that the 
high temperature phase, $\alpha-\mathrm{KAg}_{3} \mathrm{Se}_{2}$, is a superionic $\mathrm{Ag}^{+}$ion conductor, with the quasi-molten $\mathrm{Ag}$ sublattice restricted to $4 \AA$ thick layers. To the best of our knowledge, this represents the first type I superionic conductor with a 2D structure. Progressive reduction of long-range order through the melting point was probed by X-ray total scattering measurements, and the superionic local structure is also in good agreement with the AIMD simulations.

Chemical substitution has been an important tool in the design and modification of SICs; the most famous example being $\mathrm{RbAg}_{4} \mathrm{I}_{5}$, where substitution of $\mathrm{Rb}$ for $\mathrm{Ag}$ in $\mathrm{AgI}$ resulted in the highest room temperature solid-state ionic conductivity to date. ${ }^{19}$ To investigate these effects we synthesized a series of cation-substituted $A \mathrm{Ag}_{3} \mathrm{Se}_{2}(A=\mathrm{Li}-\mathrm{Cs})$ compounds. Thermal analyses indicate that the superionic transition temperature can be tuned by the composition of alkali metal ions within the immobile layers.

\section{Results}

Quasi-Elastic Neutron Scattering (QENS). QENS probes small energy transfers around a central elastic scattering peak. Dynamic processes, such as diffusion, manifest as peak broadening that grows at the expense of elastic intensity. ${ }^{20}$ The $Q$-dependence of this broadening yields information on these processes, with a linear dependence on $Q^{2}$ being indicative of translational diffusion following Fick's second law. ${ }^{21}$

Elastic scans acquired on heating from 300 to $740 \mathrm{~K}$ showed a sharp decrease in elastic intensity at $\sim 680 \mathrm{~K}$ (Figure S2 in the SI), concurrent with the $\beta-\alpha$ phase transition previously reported, ${ }^{18}$ and the on-set of quasi-elastic (QE) scattering (Figure 1a). The spectra collected of the can containing only empty fused-silica tubes overlapped with the elastic peak observed at room temperature (Figure 1b) - therefore the quasi-elastic component was from the sample only. These data were fit using delta, linear background and Lorentzian functions (Figure 1c and Section S1 in the SI), where the delta function represented purely elastic scattering and a single Lorentzian function captured the QE 
scattering component. The half-width half-maximum of this peak, $\Gamma$ was plotted as a function of $Q^{2}$ to yield information on diffusing species in the sample.

These QE scattering data were well-described by a linear fit through the origin, indicating translational Fickian diffusion in the superionic phase (see Figure 1d and Figure S3 in the SI). Similar QENS results were observed in $\alpha-\mathrm{Ag}_{2} \mathrm{Se}$, where simple Fickian diffusion was also assigned. ${ }^{22}$ In more complex jump-diffusion models, e.g., the Chudley-Elliot model, ${ }^{23}$ oscillatory behavior of $\Gamma$ occurs with increasing $Q^{2}$, associated with discrete jumps on a crystalline sublattice. Although the limited $Q$-range here means we cannot comment on the applicability of more complex models, this should not significantly affect the magnitude of the diffusion coefficient. ${ }^{24}$ The diffusion coefficient, $D$ determined from this analysis was approximately $10^{-5} \mathrm{~cm}^{2} \mathrm{~s}^{-1}$ from 710 to $740 \mathrm{~K}$, corresponding to superionic diffusion in $\alpha-\mathrm{KAg}_{3} \mathrm{Se}_{2}$. Additional QE scattering data were collected below the transition, at 600 and $650 \mathrm{~K}$, but did not exhibit features consistent with long-range diffusion (Section S1 and Figure S4 in the SI).

Interpretation of QENS data is straightforward in the case of incoherent scattering, which pertains to single particle dynamics and hence, directly to diffusion. ${ }^{21} \mathrm{Ag}$ primarily scatters neutrons coherently, with the coherent scattering cross-section being roughly an order of magnitude larger than that of the incoherent contribution (4.41 and 0.58 barns respectively). ${ }^{25}$ To account for coherency effects, the structure factor, $S(Q)$ should be considered when modelling $\Gamma(Q) \cdot{ }^{26}$ However, in QENS studies of superionic $\alpha$ - $\mathrm{Ag}_{2} \mathrm{Se}$, coherency effects were found to be negligible for $Q^{2}<2 \AA^{-2}$ (the maximum $Q^{2}$ value in our experiments), and the extracted diffusion coefficient from $D Q^{2}$ analysis matched values from ionic conductivity measurements and AIMD simulations. ${ }^{22}$ Similarly, incoherent QENS analysis of the $\mathrm{Cu}^{+}$-ion SICs ( $\mathrm{Cu}$ also mainly scatters neutrons coherently), $\alpha-\mathrm{Cu}_{2} \mathrm{Se}^{6}$ and a$\mathrm{CuCrSe}_{2}{ }^{27}$ yielded diffusion coefficient values in good agreement with conductivity measurements and molecular dynamics simulations - again suggesting minimal coherency effects. Therefore, our 
approach is reasonable based on previous results and the agreement between our incoherent $D Q^{2}$ analysis and complementary AIMD simulations.

Ab initio Molecular Dynamics (AIMD) Simulations. Molecular dynamics simulations were performed to gain insights into the superionic behavior of $\mathrm{KAg}_{3} \mathrm{Se}_{2}$. Simulations of the low temperature $\beta-\mathrm{KAg}_{3} \mathrm{Se}_{2}$ phase showed no diffusion at $300 \mathrm{~K}$, but larger displacements for $\mathrm{Ag}$ atoms (Figure S5a in the SI), consistent with the single-crystal structure solution. ${ }^{28}$ Additional simulations at 600 and $650 \mathrm{~K}$ showed no long-range diffusion of $\mathrm{Ag}^{+}$-ions in the $\beta$-phase (Figure S5b and c in the SI), consistent with the corresponding QENS experiments at these temperatures. As shown in Figure $2 \mathrm{a}$, simulations of the superionic $\alpha$-phase (space group $R-3 m$, \#166) displayed superionic $\mathrm{Ag}^{+}$ diffusion on the order of $10^{-5} \mathrm{~cm}^{2} \mathrm{~s}^{-1}$ at $800 \mathrm{~K}$, in excellent agreement with the QENS analysis. In contrast, $\mathrm{K}$ and Se did not show characteristics of diffusion and so comprise the rigid sublattice in $\alpha$ $\mathrm{KAg}_{3} \mathrm{Se}_{2}$. Mobile $\mathrm{Ag}^{+}$ions are therefore sandwiched between $\left[\mathrm{KSe}_{2}\right]^{3-}$ slabs of edge-sharing $\mathrm{KSe}_{6}$ octahedra (see Video S1 in the SI). This quasi-2D confinement is unique in the category of type I SICs. Significant similarities exist between $\alpha-\mathrm{KAg}_{3} \mathrm{Se}_{2}$ and other $2 \mathrm{D} \mathrm{Ag}^{+}$-ion SICs; especially with $\mathrm{AgCrSe}_{2}$ (type II) which shares the $R-3 m$ space group and Se anion network, but also with Ag: $\beta$ $\mathrm{Al}_{2} \mathrm{O}_{3}$ (type III) which exhibits a hexagonal ( $\mathrm{P6}_{3} / m m c$ space group, \#194) crystal structure (Figure 2b). Although we note the difference between $\mathrm{Ag}: \beta-\mathrm{Al}_{2} \mathrm{O}_{3}$, where conduction sites all lie within the same plane, and the conduction bi-layers present in $\alpha-\mathrm{KAg}_{3} \mathrm{Se}_{2}$ and $\alpha-\mathrm{AgCrSe} e_{2}$, here we define $2 \mathrm{D}$ SICs as those where long-range ionic diffusion is strongly anisotropic, i.e., there is negligible ionic conductivity perpendicular to the layers.

Additional AIMD simulations were performed at 700 and $900 \mathrm{~K}$ to obtain data points for activation energy analysis (Figure S6 in the SI). Simple fitting of $D$ as a function of temperature was performed using an Arrhenius relationship,

$D(T)=D_{0} \exp \left(-E_{a} / k_{B} T\right)$ 
where, $D_{0}$ is a model-dependent pre-exponential factor, $E_{a}$ is the activation energy for diffusion, $k_{B}$ is the Boltzmann constant and $T$ is temperature. Application of equation (1) yielded an $E_{a, A I M D}=$ $0.013(2) \mathrm{eV}$ and $D_{0, A I M D}=3.47(1) \times 10^{-5} \mathrm{~cm}^{2} \mathrm{~s}^{-1}$. The small $E_{a}$ value, on the order of $k_{B} T$, was consistent with facile ionic diffusion and that measured for 3D type I SICs such as $\mathrm{AgI},{ }^{29} \mathrm{Ag}_{2} \mathrm{~S}^{30}$ and $\mathrm{Ag}_{2} \mathrm{Se}^{31,32}$ The experimental QENS data are consistent with the trend over the wider $T$ range from simulations (Figure 2c).

Pair-Distribution-Function Analysis. Analyses of the local structures of the $\beta, \alpha$ and liquid phases of $\mathrm{KAg}_{3} \mathrm{Se}_{2}$ were achieved using X-ray pair-distribution-function (PDF) analysis. In the superionic $\alpha$-phase, atomic correlations were broader and weaker in magnitude than in the ordered $\beta$-phase (Figure 3a). Liquid $\mathrm{KAg}_{3} \mathrm{Se}_{2}$ closely resembled the nearest-neighbor Ag-Se, Se-Se and $\mathrm{Ag}-\mathrm{Ag}$ correlations in liquid $\mathrm{Ag}_{2} \mathrm{Se}^{33}$

The local structures of both $\beta$ - and $\alpha-\mathrm{KAg}_{3} \mathrm{Se}_{2}$ were well-described using the previously reported average structures and refining anisotropic atomic displacement parameters (ADPs). Weighted residuals $\left(R_{w}\right)$ of $\sim 10 \%$ and $\sim 20 \%$ were achieved in the ordered $\beta$-phase and disordered $\alpha$-phase respectively (Figure S7 in the SI). Full fitting details can be located in Section S2 in the SI. PDF analysis has been shown to produce reliable ADPs, comparable to those from single-crystal refinement. ${ }^{34}$ Indeed, for the low temperature phase, the anisotropic ADPs were in good agreement with those from single-crystal data (Figure S8 in the SI). Three unique Ag atoms describe the corrugated $\left[\mathrm{Ag}_{3} \mathrm{Se}_{2}\right]^{-}$layers, with $\mathrm{Ag} 1$ and $\mathrm{Ag} 3$ forming tunnels along the $b$-axis, which are bridged by Ag2 (Figure 3b). The type I superionic phase transition was observed around $700 \mathrm{~K}$, fully occurring in a $\sim 15 \mathrm{~K}$ window (Figure $\mathrm{S} 9$ in the SI). As shown in Figure 3b, the Ag ADPs became very large (all with $U_{e q}$ of $\sim 0.1 \AA^{2}$ ) and anisotropic as the transition was approached, consistent with the vibrations of these atoms, in particular $\mathrm{Ag} 2$, destabilising the ordered phase and driving the transition to the superionic state (see Section S2 in the SI). 
In superionic a- $\mathrm{KAg}_{3} \mathrm{Se}_{2}$, the silver layers are comprised of two unique sites with $75 \%$ occupancy: $\mathrm{Ag} 1$ in a bent trigonal planar and Ag2 in a distorted tetrahedral coordination (Figure $3 \mathrm{~b}$ ). Both Ag sites are characterized by large displacement parameters which can indicate static or dynamic disorder. As shown in Figure 3c, the Ag probability densities derived from AIMD simulations qualitatively match the ADPs from PDF analysis, suggesting that the Ag ions are dynamically disordered - a common feature of SICs. Our simulations indicated no strong preference between $\mathrm{Ag} 1$ or $\mathrm{Ag} 2$ sites on the timescales probed here and that all $\mathrm{Ag}^{+}$ions were mobile, thus an in-plane ionic conductivity, $\sigma_{I}=n e D Z / k_{B} T$, of $\sim 0.5 \mathrm{~S} \mathrm{~cm}^{-1}$ could be estimated at $800 \mathrm{~K}$. In contrast, probability distributions for $\mathrm{K}$ and Se showed localised and isotropic distributions, reflecting harmonic potentials (Figure S11 in the SI).

Cation Substitution. As shown in the preceding section, strongly anisotropic Ag thermal motion appears to drive the superionic transition with seemingly little contribution from the chargebalancing K-Se layers. Substitutions of $\mathrm{K}^{+}$with $\mathrm{Na}^{+}, \mathrm{Rb}^{+}$and $\mathrm{Cs}^{+}$in the ordered $\beta$-phase of $\mathrm{KAg}_{3} \mathrm{Se}_{2}$ were accomplished by solid-state methods and the solid-transition onset temperatures, $T_{\text {solid }}$, were monitored using DTA (Figures 4 and S12 in the SI). Attempts at full replacement of $\mathrm{K}^{+}$with $\mathrm{Li}^{+}$or $\mathrm{Na}^{+}$ions resulted in no ternary formation or a new, unknown ternary phase respectively (see Figure $\mathrm{S} 14$ in the SI), while neither $\mathrm{RbAg}_{3} \mathrm{Se}_{2}$ nor $\mathrm{CsAg}_{3} \mathrm{Se}_{2}$ exhibited features consistent with a solid phase transition before melting (Figure 4a).

The lack of sharp transitions in the latter compounds was somewhat unexpected. Both $\mathrm{RbAg}_{3} \mathrm{Se}_{2}$ and $\mathrm{CsAg}_{3} \mathrm{Se}_{2}$ are isostructural with $\beta-\mathrm{KAg}_{3} \mathrm{Se}_{2}$, with virtually no change in $\mathrm{Ag}$-Se interatomic distances $(<0.01 \AA),{ }^{28}$ suggesting the underlying mechanism is not simply due to "chemical pressure" effects. To explore this further, a series of samples with compositions, $A_{x} \mathrm{~K}_{1-x} \mathrm{Ag}_{3} \mathrm{Se}_{2}(A=\mathrm{Na}, \mathrm{Rb})$ were synthesized and characterised. A complete solid-solution was possible between $\mathrm{KAg}_{3} \mathrm{Se}_{2}$ and $\mathrm{RbAg}_{3} \mathrm{Se}_{2}$, while $\mathrm{Na}_{x} \mathrm{~K}_{1-x} \mathrm{Ag}_{3} \mathrm{Se}_{2}$ was limited to $x=0.1$, above which significant impurities were observed by PXRD (Figure S15 in the SI). Alternative synthetic approaches such as ion exchange, 
flux or thin-film synthesis may be successful in realising compounds with increased Na content. The $T_{\text {solid }}$ values for these series are shown in Figure $4 \mathrm{~b}$. When smaller ions were substituted for $\mathrm{K}$, the $T_{\text {solid }}$ decreased and the hysteresis between transitions on heating (endothermic) and cooling (exothermic) increased. Conversely, when $\mathrm{Rb}$ replaced $\mathrm{K}$ in the immobile, charge-balancing layers, the solid transition was pushed to higher temperatures with negligible hysteresis for members with $x$ $\geq 0.25$. Comparing the $T_{\text {solid }}$ values of the most Na-rich and Rb-rich compounds synthesised, chemical control of the solid transition of over $100 \mathrm{~K}$ is demonstrated. Taken altogether, we speculate that the larger alkali metal ions are less able to adopt the perfect octahedral geometry with Se observed in $\alpha-\mathrm{KAg}_{3} \mathrm{Se}_{2}$ (Figures $2 \mathrm{~b}$ and $3 \mathrm{c}$ ), inhibiting the superionic transition and vice versa. This approach to modulating the superionic transition temperature is distinct from the nanoparticle size effects observed in $\mathrm{AgI}^{35}$ or the effects of applied pressure and can be readily explored in bulk materials. However, further characterisation (e.g., in-situ X-ray ray scattering, differential scanning calorimetry, QENS) of additional members of these solid solutions $A_{x} \mathrm{~K}_{1-x} \mathrm{Ag}_{3} \mathrm{Se}_{2}$, where $A=$ alkali metals will be required to definitively establish this trend and will be the focus of future work.

\section{Conclusions}

$\alpha-\mathrm{KAg}_{3} \mathrm{Se}_{2}$ is a new type I superionic conductor and the first with a 2D crystal structure. Quasielastic neutron scattering (QENS) between 710 and $740 \mathrm{~K}$ yields a diffusion coefficient of $\sim 10^{-5} \mathrm{~cm}^{2}$ $\mathrm{s}^{-1}$, comparable to that of liquid water at room temperature. Our ab initio molecular dynamics (AIMD) simulations show that $\mathrm{K}$ and Se atoms form the immobile sublattice and the superionic $\mathrm{Ag}^{+}$ ions reside in $4 \AA$ thick infinite slabs. The AIMD simulations reproduce the diffusion coefficient from QENS and the local structure measured by powder X-ray pair-distribution-function analysis. Initial exploration of isostructural analogues suggests that, rather than any chemical pressure effects on the Ag-Se network, the malleability of the charge-balancing ions facilitates or inhibits the superionic phase transition temperature. The availability of this compound opens a new stage for experimentation aimed at studying in detail ionic motion confined in two dimensions. Our work 
extends the known classes of superionic conductors and should facilitate the design of new materials with tailored ionic conductivities and phase transitions.

\section{Methods}

Reagents. The following reagents were used as received: sodium metal (99.9\%, Sigma-Aldrich), potassium metal (99\%, Sigma-Aldrich), rubidium metal (99\%, Johnson Matthey) caesium metal (99.8\%, Sigma-Aldrich), silver shot (99.99\%, Alfa Aesar) and selenium shot (99.999\%, Plasmaterials Inc.).

Synthesis. All chemical manipulations were conducted inside an Ar-filled glovebox (M-Braun) with oxygen and moisture levels $<0.1 \mathrm{ppm}$. Flame-sealing was performed by evacuating ampules to $<10^{-}$ ${ }^{3}$ mbar. Phase-pure $\mathrm{KAg}_{3} \mathrm{Se}_{2}$ powder was synthesised directly from the elements, with $\mathrm{K}$ and $\mathrm{Se}$ physically separated by Ag shot to avoid a strongly exothermic reaction. Elemental ratios K:Ag:Se of 1.05:3:2 were used, with a 5\% molar excess $\mathrm{K}$ to account for surface oxidation of the metal. In a typical experiment, $\mathrm{K}$ metal chunks $(1.101 \mathrm{~g}, 28 \mathrm{mmol})$ were compacted in the bottom of an $18 \mathrm{~mm}$ O.D. (outer diameter) $\times 16 \mathrm{~mm}$ I.D. (inner diameter) fused-silica tube. Ag shot $(8.682 \mathrm{~g}, 80 \mathrm{mmol})$ was then loaded followed by Se shot $(4.237 \mathrm{~g}, 54 \mathrm{mmol})$ on top. To minimize the free volume inside the ampule, a $15 \mathrm{~mm}$ O.D. $\times 13 \mathrm{~mm}$ I.D. fused-silica tube was used as a snug-fitting plug above the elements. This assembly was then flame-sealed and placed upright in a box furnace. The mixture was heated to $400{ }^{\circ} \mathrm{C}$ in $12 \mathrm{~h}$ and held for $12 \mathrm{~h}$ after which the furnace was turned off.

$A_{2} \mathrm{Se}_{3}(A=\mathrm{Rb}, \mathrm{Cs})$ precursor powders were synthesized using a tube-in-tube method. Briefly, a stoichiometric amount of Se was suspended in an alumina crucible above $\mathrm{Rb}$ or Cs metal and flamesealed in an $18 \mathrm{~mm}$ O.D. $\times 16 \mathrm{~mm}$ I.D. fused-silica tube while the bottom of the tube was submerged in liquid nitrogen. This assembly was placed upright in a muffle furnace and heated to $500{ }^{\circ} \mathrm{C}$ in $20 \mathrm{~h}$ and held at that temperature for $12 \mathrm{~h}$ before natural cooling to room temperature. A black $A_{2} \mathrm{Se}_{3}$ ingot could be removed from the bottom of the tube in both cases. $\mathrm{Li}_{2} \mathrm{Se}$ and $\mathrm{Na}_{2} \mathrm{Se}$ were 
produced by combining the elements in a 2:1 molar ratio in liquid ammonia using established procedures. $^{36}$

To synthesise the $\mathrm{RbAg}_{3} \mathrm{Se}_{2}$ and $\mathrm{CsAg}_{3} \mathrm{Se}_{2}$ ternary compounds, $A_{2} \mathrm{Se}_{3}(A=\mathrm{Rb}, \mathrm{Cs}), \mathrm{Ag}$ and $\mathrm{Se}$ powders were homogenized in a mortar and pestle in a 1:6:1 molar ratio, loaded into a graphite crucible and capped with a graphite plug before being flame-sealed in a $15 \mathrm{~mm}$ O.D. $\times 13 \mathrm{~mm}$ I.D. fused-silica tube. These mixtures were heated to $400{ }^{\circ} \mathrm{C}$ in $3 \mathrm{~h}$ and held for $12 \mathrm{~h}$ before the furnace was turned off. Similar procedures were used for targeting $\mathrm{LiAg}_{3} \mathrm{Se}_{2}, \mathrm{Na}_{x} \mathrm{~K}_{1-x} \mathrm{Ag}_{3} \mathrm{Se}_{2}(x=0.05,0.01$, $0.25,1)$ and $\mathrm{Rb}_{x} \mathrm{~K}_{1-x} \mathrm{Ag}_{3} \mathrm{Se}_{2}(x=0.05,0.01,0.25,0.75)$ using stoichiometric molar ratios of $\mathrm{Li}_{2} \mathrm{Se}$, $\mathrm{Na}_{2} \mathrm{Se}, \mathrm{Ag}, \mathrm{Se}, \mathrm{KAg}_{3} \mathrm{Se}_{2}$ and $\mathrm{RbAg}_{3} \mathrm{Se}_{2}$. In all cases a black, homogeneous powder compact was produced. Powder X-ray diffractograms for all products can be located in the Supporting Information (SI, Figures S13 and 14).

Quasi-Elastic Neutron Scattering. Quasi-elastic neutron scattering (QENS) data were collected at the backscattering silicon spectrometer (BASIS) ${ }^{37}$ at the Spallation Neutron Source, Oak Ridge National Laboratory (ORNL) using a Closed Cycle Refrigerator sample environment (maximum operating temperature: $740 \mathrm{~K}$ ). Because of the high absorption cross-section of Ag, $\sim 10 \mathrm{~g}$ of $\mathrm{KAg}_{3} \mathrm{Se}_{2}$ was loaded into four $7 \mathrm{~mm}$ O.D. $\times 6 \mathrm{~mm}$ I.D. fused-silica tubes and flame-sealed. These were placed side-by-side in the Al sample can to approximate a flat plate scattering geometry (see Figure S16 in the SI). Furnaces capable of higher temperature operation had much larger background scattering from Al and so were not suitable. First, short elastic scans from 300 to $740 \mathrm{~K}$ were measured. A change in elastic intensity around $680 \mathrm{~K}$ was assigned to the known order-disorder phase transition, albeit at a lower temperature than $695 \mathrm{~K}$ observed by differential scanning calorimetry (DSC). DSC measurements are thoroughly calibrated; thus, this discrepancy was attributed to poor sample-thermocouple coupling in the QENS sample environment. Spectra were then collected on cooling between 740 and $710 \mathrm{~K}$ in $10 \mathrm{~K}$ increments, followed by a long QENS measurements at $300 \mathrm{~K}$, which was used as the instrument resolution function. Finally, four 
nominally identical, empty, evacuated tubes were used for the background measurement at $740 \mathrm{~K}$. Additional long QENS experiments were performed at 600 and $650 \mathrm{~K}$. These data were collected on heating from room temperature to ensure the sample was in the $\beta$-phase. $S(Q, \omega)$ data were reduced using Mantid software ${ }^{38}$ in the range -100 to $100 \mathrm{meV}$ and binned in $0.04 \mathrm{meV}$ steps over a $Q$ range of 0.3-1.3 $\AA^{-1}$ binned in $0.1 \AA^{-1}$ steps. Fitting of the QENS data (see Section S1 in SI) was performed using the Data Analysis and Visualization Environment (DAVE) package. ${ }^{38}$ The bandwidth of incoming neutron was centered at $6.4 \AA$. Coherent scattering from Bragg peaks was present between $Q^{2}=0.5-0.64 \AA^{-2}$ and $Q^{2}>1.9 \AA^{-2}$, thus these $Q$-ranges were excluded from the analysis (Figure S14 in the SI). ${ }^{20}$ However, we note that the inclusion of these data did not markedly affect the fitting results.

Ab initio Molecular Dynamics Calculations. Ab initio molecular dynamics (AIMD) simulations were performed using the Vienna Ab initio Simulation Package (VASP), ${ }^{39-41}$ using as exchangecorrelation functional the generalized gradient approximation (GGA) in the Perdew-BurkeErnzerhof (PBE) form. ${ }^{42,43}$ Convergence tests were conducted with low temperature $\beta-\mathrm{KAg}_{3} \mathrm{Se}_{2}$, where a plane-wave cut-off energy of $350 \mathrm{eV}$ and an electronic $k$-point mesh of $3 \times 12 \times 6$ were used. The electronic self-consistent loop converged to $10^{-8} \mathrm{eV}$ and the forces were smaller than $10^{-4}$ $\mathrm{eV} / \AA ̊$ on all atoms. AIMD simulations were performed on a $4 \times 4 \times 1$ supercell of the superionic $\alpha$ $\mathrm{KAg}_{3} \mathrm{Se}_{2}$ (288 atoms) using the experimental lattice constants, $a=4.56 \AA$, and $\mathrm{c}=25.41 \AA$, with the Brillouin zone center, $\mathrm{G}$, as the only $k$-point. Three parallel trajectories starting with random initial structures were calculated at 700, 800, and $900 \mathrm{~K}$, and averaged for mean square displacement MSD calculations. For each initial structure, the stoichiometry (75\% Ag site occupancy) was maintained by randomly removing $25 \%$ of $\mathrm{Ag}$ atoms in each layer. Additional simulations at 300, 600 and 650 $\mathrm{K}$ were conducted for the $\beta$-phase. Each trajectory was at least 55 ps long with 2 fs steps, and the first 5 ps were treated as equilibration time. Extraction of the diffusion coefficient was performed by linear fitting between the bounds of $0.5 a^{2}$, where $a$ is the jump distance, $\left(\sim 4.5 \mathrm{~A}^{2}\right)$ and $70 \%$ of the simulation time (35 ps) as recommended by He et al. ${ }^{44}$ 
Synchrotron X-ray Scattering. In-situ X-ray total scattering data were collected on heating at Sector 17-BM-B at the Advanced Photon Source from 300 to $950 \mathrm{~K}$ using a PerkinElmer amorphous silicon flat panel detector. $\mathrm{KAg}_{3} \mathrm{Se}_{2}$ powder was sieved to $<45 \mu \mathrm{m}$ and loaded into a $0.3 \mathrm{~mm}$ diameter fused-silica capillary and flame-sealed. The sample was spun during collection and the wavelength used was $0.24141 \AA$. Integration and background subtraction was performed using GSAS-II software (version 3667), ${ }^{45}$ transformation from $S(Q)$ to $G(r)$ using PDFgetX3 ${ }^{46}$ (with $Q_{\max }$ $=18 \AA^{-1}$ ) and fitting using pdfgui software. ${ }^{47}$

Powder X-ray Diffraction. Phase purity was determined by powder X-ray diffraction (PXRD). Data were collected using a Rigaku Miniflex diffractometer with a Ni-filtered $\mathrm{Cu} \mathrm{Ka}$ source operating at $15 \mathrm{~mA}$ and $40 \mathrm{kV}$. Continuous scanning was utilized with a step size of $0.015^{\circ}$. Materials were finely ground and uniformly coated on a flat plate sample holder and protected from ambient conditions using Kapton film and vacuum grease.

Differential Thermal Analysis. Differential thermal analyses (DTA) were carried out using a Shimadzu DTA-50 thermal analyser. The powder samples ( 30 mg total mass) were evacuated and sealed in carbon-coated, fused-silica ampules. A fused-silica ampule containing alumina powder was used as a reference. All samples were heated to the desired temperature at $5 \mathrm{~K} \mathrm{~min}^{-1}$, followed by cooling at the same rate to $343 \mathrm{~K}$. Transition temperatures were determined from the onset of endothermic or exothermic features.

\section{Acknowledgements}

A.R. acknowledges Wenqian Xu for assistance in acquisition and analysis of X-ray total scattering data. This work was performed primarily at the Materials Science Division at Argonne National Laboratory supported by the U.S. Department of Energy, Office of Science, Office of Basic Energy Sciences, Materials Sciences and Engineering Division. We gratefully acknowledge the computing 
resources provided on Bebop; the high-performance computing clusters operated by the Laboratory Computing Resource Center at Argonne National Laboratory. First-principles modeling at Duke University (J.D., O.D.) was supported by the U.S. Department of Energy, Office of Science, Basic Energy Sciences, Materials Sciences and Engineering Division, under Award No. DE-SC0019299. Work at ORNL's Spallation Neutron Source is supported by the U.S. Department of Energy, Office of Basic Energy Sciences. The Oak Ridge National Laboratory is managed by UT-Battelle, LLC, for U.S. DOE under Contract No. DEAC05-00OR22725.

\section{Author Contributions}

A.R., S.R. and M.G.K. conceived the study. A.R., X.Z. and D.-Y.C. synthesised and characterised all materials. A.R., R.O. and N.O. acquired and analysed neutron scattering data. J.D. and O.D. performed AIMD simulations. M.J. and C.M. conducted PDF analysis and structural modelling. The manuscript was mainly written and revised by A.R., S.R. and M.G.K. All authors approved the final version of the manuscript.

\section{Competing Interests Statement}

The authors declare no competing interests.

\section{Associated Content}

\section{Supplementary Information}

Experimental details of QENS measurements, additional AIMD simulations, PDF analysis, powder XRD of $A \mathrm{Ag}_{3} \mathrm{Se}_{2}(A=\mathrm{Li}-\mathrm{Cs})$ compounds and DTA data.

\section{References}

1 Boyce, J. B. \& Huberman, B. A. Superionic conductors: Transitions, structures, dynamics. Phys. Rep. 51, 189-265 (1979).

2 Faraday, M. VII. Experimental researches in electricity. Phil. Trans. R. Soc 128, 83-123 (1838).

3 Hull, S. Superionics: crystal structures and conduction processes. Rep. Prog. Phys 67, 12331314 (2004). 
Goodenough, J. B. Review Lecture-Fast ionic conduction in solid. Proc. R. Soc. A 393, 215234 (1984).

$5 \quad$ Bruce, P. G. Solid State Electrochemistry. (Cambridge University Press, 1997).

6 Voneshen, D., Walker, H., Refson, K. \& Goff, J. Hopping time scales and the phonon-liquid electron-crystal picture in thermoelectric copper selenide. Phys. Rev. Lett. 118, 145901 (2017).

7 Ding, J. et al. Anharmonic lattice dynamics and superionic transition in $\mathrm{AgCrSe}_{2}$. Proc. Natl. Acad. Sci USA 117, 3930-3937 (2020).

8 Bailey, T. P. \& Uher, C. Potential for superionic conductors in thermoelectric applications. Curr. Opin. Green Sustainable Chem 4, 58-63 (2017).

9 Keen, D. A. Disordering phenomena in superionic conductors. J. Condens. Matter Phys. 14, R819 (2002).

10 Funke, K. AgI-type solid electrolytes. Prog. Solid. State Ch. 11, 345-402 (1976).

11 Derrington, C. \& O'Keeffe, M. Anion conductivity and disorder in lead fluoride. Nat. Phys. Sci. 246, 44-46 (1973).

Boukamp, B. \& Wiegers, G. Ionic and electronic processes in $\mathrm{AgCrSe}$. Solid State Ion. 9 , 1193-1196 (1983).

Yao, Y.-F. Y. \& Kummer, J. Ion exchange properties of and rates of ionic diffusion in betaalumina. J. Inorg. Nucl. Chem. 29, 2453IN12467-24662475 (1967).

Engelsman, F., Wiegers, G., Jellinek, F. \& Van Laar, B. Crystal structures and magnetic structures of some metal (I) chromium (III) sulfides and selenides. J. Solid State Chem. 6 , 574-582 (1973).

15 Newsam, J. \& Cheetham, A. Stoichiometric silver beta alumina studied at 25, 300 and 500 degrees C by powder neutron diffraction. J. Phys. Condens. Matter 2, 2335 (1990). Tubandt, C. \& Lorenz, E. Molekularzustand und elektrisches Leitvermögen kristallisierter Salze. Z. Phys. Chem 87, 513-542 (1914). Miyatani, S.-y. Ionic conductivity in silver chalcogenides. J. Phys. Soc. Jpn 50, 3415-3418 (1981).

Rettie, A. J. E. et al. $\mathrm{Ag}_{2} \mathrm{Se}$ to $\mathrm{KAg}_{3} \mathrm{Se}_{2}$ : Suppressing Order-Disorder Transitions via Reduced Dimensionality. J. Am. Chem. Soc. 140, 9193-9202 (2018). Raleigh, D. O. Ionic conductivity of single-crystal and polycrystalline $\mathrm{RbAg}_{4} \mathrm{I}_{5}$. J. Appl. Phys. 41, 1876-1877 (1970).

20 Mamontov, E. Fast oxygen diffusion in bismuth oxide probed by quasielastic neutron scattering. Solid State Ion. 296, 158-162 (2016).

21 Bée, M. Localized and long-range diffusion in condensed matter: state of the art of QENS studies and future prospects. Chem. Phys. 292, 121-141 (2003). Hamilton, M., Barnes, A., Howells, W. \& Fischer, $\mathrm{H} . ~_{\mathrm{Ag}^{+}}$dynamics in the superionic and liquid phases of $\mathrm{Ag}_{2} \mathrm{Se}$ and $\mathrm{Ag}_{2} \mathrm{Te}$ by coherent quasi-elastic neutron scattering. J. Phys. Condens. Matter 13, 2425 (2001).

Chudley, C. \& Elliott, R. Neutron scattering from a liquid on a jump diffusion model. Proc. Phys. Soc. 77, 353 (1961).

Embs, J. P., Juranyi, F. \& Hempelmann, R. Introduction to quasielastic neutron scattering. Z. Phys. Chem 224, 5-32 (2010).

Hempelmann, R. Quasielastic neutron scattering and solid state diffusion. (Clarendon Press, 2000).

Wind, J., Mole, R. A., Yu, D. \& Ling, C. D. Liquid-like ionic diffusion in solid bismuth oxide revealed by coherent quasielastic neutron scattering. Chem. Mater. 29, 7408-7415 (2017).

Delaire, O. et al. Selective breakdown of phonon quasiparticles across superionic transition in CuCrSe 2. APS 2019, R47. 002 (2019).

Bensch, W. \& Dürichen, P. Crystal structure of potassium diselenotriargentate, $\mathrm{KAg}_{3} \mathrm{Se}_{2}$. Z. Kristallogr. - New Cryst. Struct. 212, 97-98 (1997).

Kvist, A. \& Josefson, A.-M. The electrical conductivity of solid and molten silver iodide. $Z$. Naturforsch. A 23, 625-626 (1968). 
Allen, R. L. \& Moore, W. J. Diffusion of silver in silver sulfide. J. Phys. Chem. 63, 223-226 (1959).

31 Okazaki, H. Deviation from the Einstein relation in average crystals self-diffusion of $\mathrm{Ag}^{+}$ ions in $\alpha-\mathrm{Ag}_{2} \mathrm{~S}$ and $\alpha-\mathrm{Ag}_{2} \mathrm{Se}$. J. Phys. Soc. Jpn 23, 355-360 (1967).

32 Rom, I. \& Sitte, W. Composition dependent ionic and electronic conductivities and chemical diffusion coefficient of silver selenide at 160 C. Solid State Ion. 101, 381-386 (1997).

33 Barnes, A., Lague, S., Salmon, P. \& Fischer, H. A determination of the structure of liquid $\mathrm{Ag}_{2} \mathrm{Se}$ using neutron diffraction and isotopic substitution. J. Phys. Condens. Matter 9, 6159 (1997).

34 Lee, S. \& Xu, H. Using complementary methods of synchrotron radiation powder diffraction and pair distribution function to refine crystal structures with high quality parameters - A Review. Minerals 10, 124 (2020).

Makiura, R. et al. Size-controlled stabilization of the superionic phase to room temperature in polymer-coated AgI nanoparticles. Nat. Mater. 8, 476-480 (2009).

36 Sharp, K. W. \& Koehler, W. H. Synthesis and characterization of sodium polyselenides in liquid ammonia solution. Inorg. Chem. 16, 2258-2265 (1977).

37 Mamontov, E. \& Herwig, K. W. A time-of-flight backscattering spectrometer at the Spallation Neutron Source, BASIS. Rev. Sci. Instrum. 82, 085109 (2011).

38 Arnold, O. et al. Mantid-Data analysis and visualization package for neutron scattering and $\mu$ SR experiments. Nucl. Instrum. Methods Phys. Res. A 764, 156-166 (2014).

39 Kresse, G. \& Furthmüller, J. Efficient iterative schemes for ab initio total-energy calculations using a plane-wave basis set. Phys. Rev. B 54, 11169 (1996).

40 Kresse, G. \& Furthmüller, J. Efficiency of ab-initio total energy calculations for metals and semiconductors using a plane-wave basis set. Comput. Mater. Sci. 6, 15-50 (1996).

41 Kresse, G. \& Hafner, J. Ab initio molecular dynamics for liquid metals. Phys. Rev. B 47, 558 (1993).

42 Perdew, J. P., Burke, K. \& Ernzerhof, M. Generalized gradient approximation made simple. Phys. Rev. Lett. 77, 3865-3868 (1996).

43 Perdew, J. P. \& Zunger, A. Self-interaction correction to density-functional approximations for many-electron systems. Phys. Rev. B 23, 5048 (1981).

44 He, X., Zhu, Y., Epstein, A. \& Mo, Y. Statistical variances of diffusional properties from ab initio molecular dynamics simulations. Npj Computational Materials 4, 1-9 (2018).

45 Toby, B. H. \& Von Dreele, R. B. GSAS-II: the genesis of a modern open-source all purpose crystallography software package. J. Appl. Crystallogr. 46, 544-549 (2013).

46 Juhás, P., Davis, T., Farrow, C. L. \& Billinge, S. J. L. PDFgetX3: a rapid and highly automatable program for processing powder diffraction data into total scattering pair distribution functions. J. Appl. Crystallogr. 46, 560-566 (2013).

47 Farrow, C. L. et al. PDFfit2 and PDFgui: computer programs for studying nanostructure in crystals. J. Phys. Condens. Matter 19, 335219 (2007).

\section{Figures and Captions}



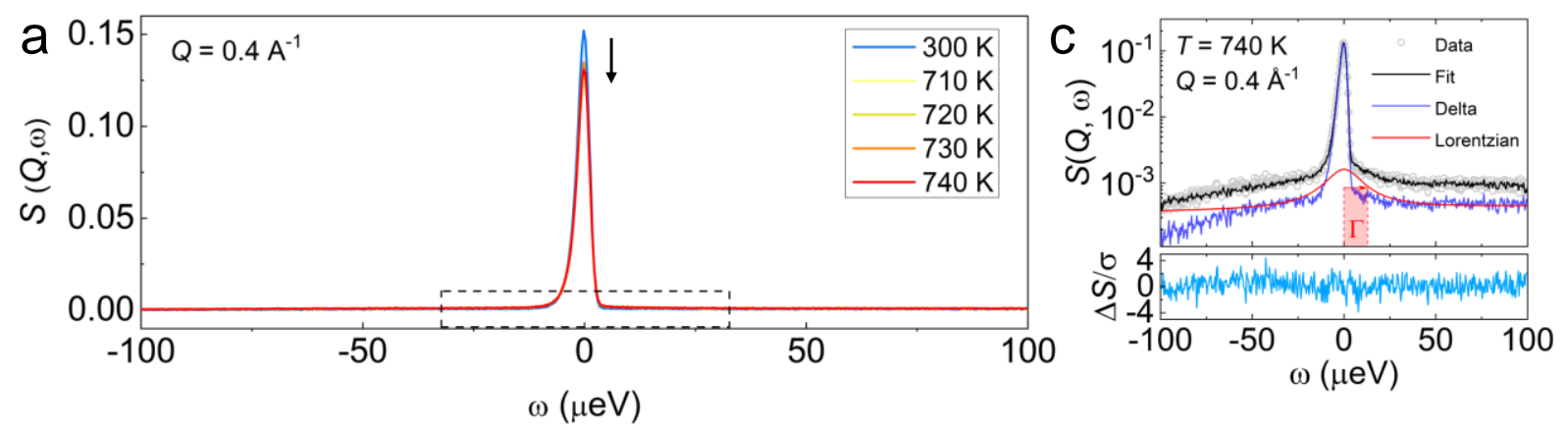

b
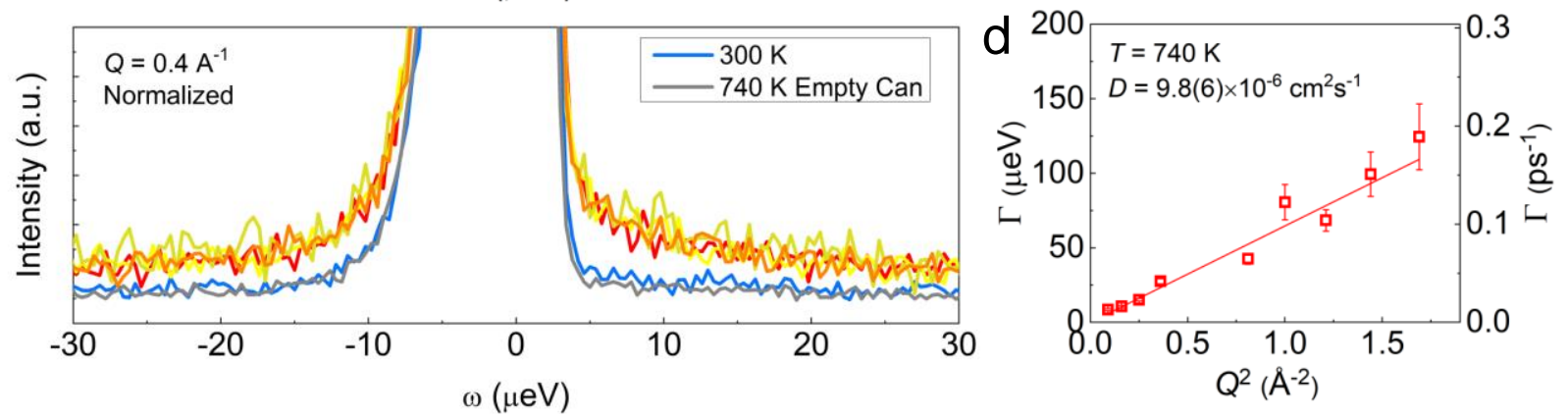

Figure 1. a) Representative QENS spectra at $Q=0.4 \AA^{-1}$ as a function of temperature for $\mathrm{KAg}_{3} \mathrm{Se}_{2}$. The arrow shows a sharp decrease in elastic intensity for the high temperature $\alpha$-phase. $\mathbf{b})$ Normalised $S(Q, \omega)$ from the boxed region in b), illustrating quasi-elastic broadening in $\alpha-\mathrm{KAg}_{3} \mathrm{Se}_{2}$. The legend is the same as A). c) Fitting of $S(Q, \omega)$ at $740 \mathrm{~K}$ and $Q=0.4 \AA^{-1}$ using linear background (BG), delta and Lorentzian functions. Note that the BG function has been added to the Lorentzian component for clarity. d) Half-width half-maximum of the Lorentzian component, $\Gamma$ vs. $Q^{2}$. A linear fit yielded the diffusion coefficient, D. $Q$-regions that include strong Bragg contributions have been omitted. 

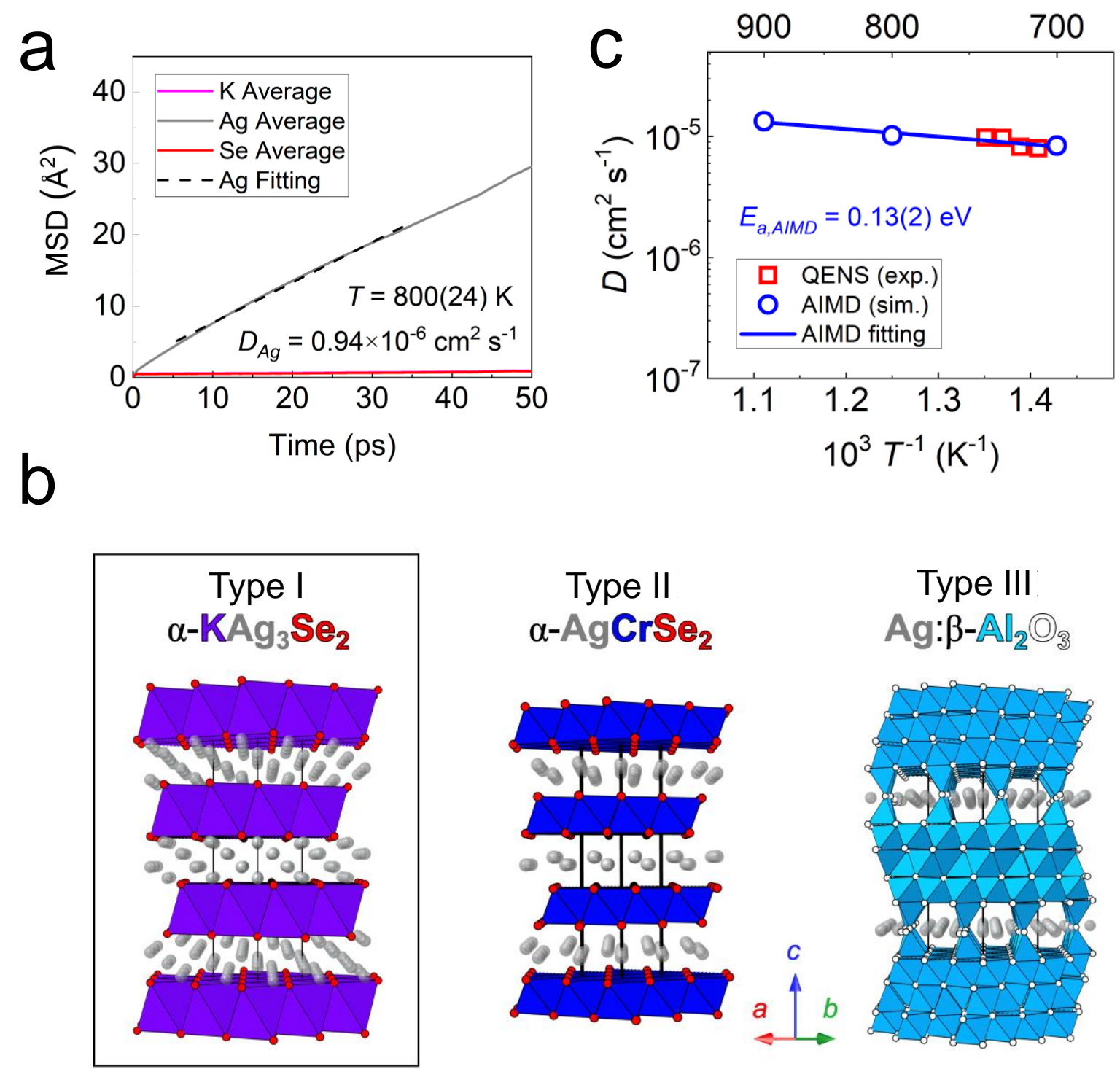

Figure 2. a) AIMD simulated mean square displacement (MSD) of atomic species in $\alpha-\mathrm{KAg}_{3} \mathrm{Se}_{2}$ at $800 \mathrm{~K}$. The linear fit yields the diffusion coefficient of Ag ions. b) Crystal structures of 2D $\mathrm{Ag}^{+}$ion SICs organized by type. The immobile rigid lattice is represented by solid polyhedra and the mobile $\mathrm{Ag}^{+}$ions as translucent spheres. The unit cells are drawn with solid black lines. c) Extracted diffusion coefficients from QENS (open red squares) and those derived from AIMD simulations (open blue circles) vs. reciprocal temperature. Error bars have been omitted for clarity but were used in the determination of the AIMD activation energy (linear fit represented by a solid blue line). 


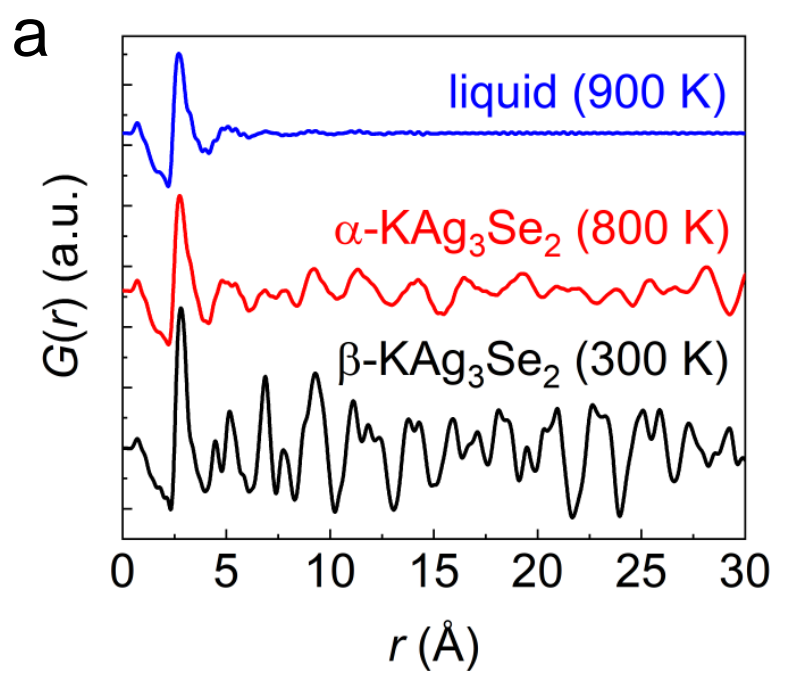

b

\section{$\left[\AA \mathrm{Ag}_{3} \mathrm{Se}_{2}\right]^{\text {-layer }}$}
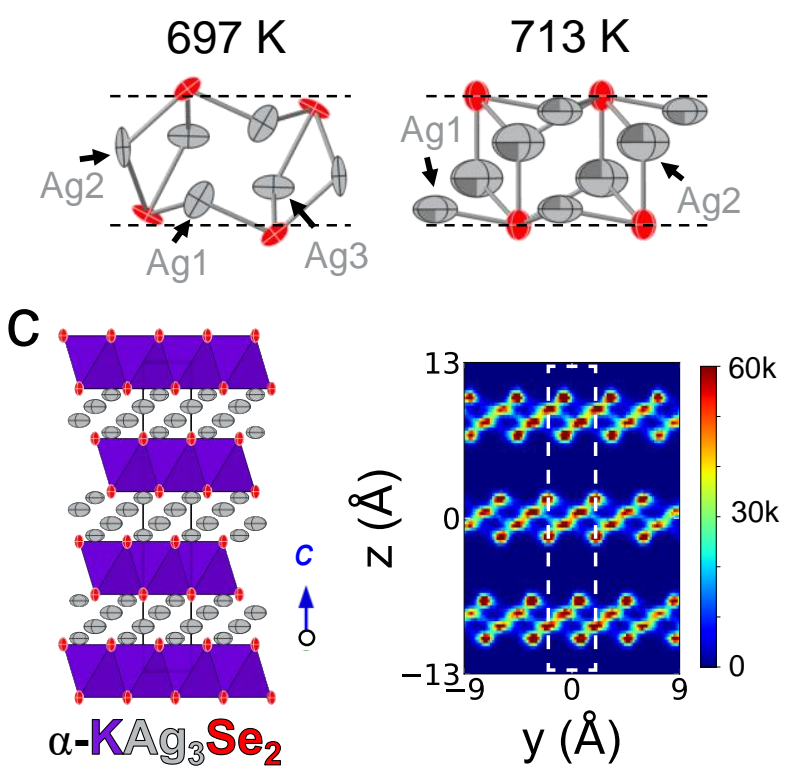

Figure 3. a) In-situ X-ray PDF of $\mathrm{KAg}_{3} \mathrm{Se}_{2}$ powder. b) Refined local structures of the $\mathrm{Ag}$-Se layers just below $(697 \mathrm{~K})$ and just above $(713 \mathrm{~K})$ the superionic transition, in the $\beta$ - and $\alpha$-phases respectively. Note that the $\mathrm{Ag}$ atoms at $713 \mathrm{~K}$ ( $\alpha$-phase) have $75 \%$ occupancy. c) Crystal structure of $\alpha-\mathrm{KAg}_{3} \mathrm{Se}_{2}$ from PDF analysis (left) compared with simulated Ag probability densities, both at 800 $\mathrm{K}$ (right). The unit cell is shown by solid black and dashed white lines respectively. Thermal ellipsoids are shown at $50 \%$. 

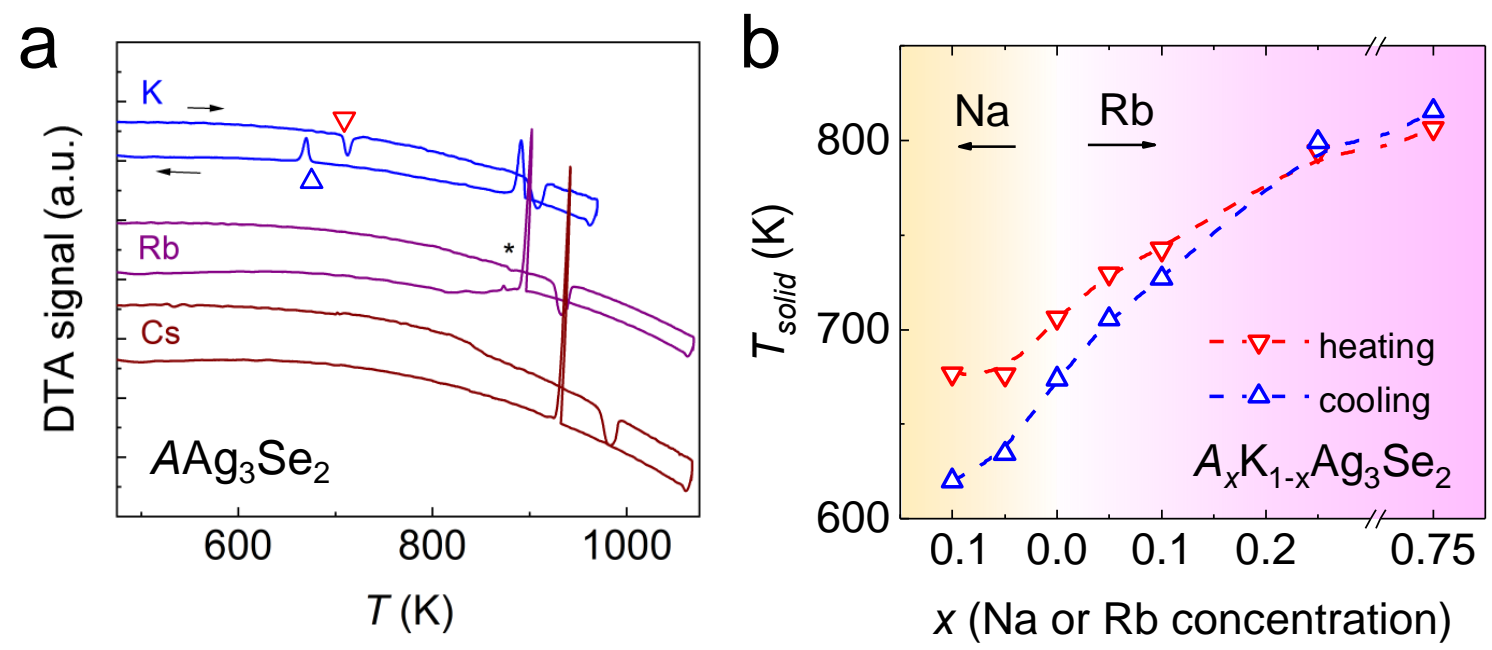

Figure 4. a) DTA curves for $\mathrm{AAg}_{3} \mathrm{Se}_{2}(A=\mathrm{K}-\mathrm{Cs})$ illustrating the effect of cation substitution on endo/exothermic (red down triangle/blue up triangle symbols respectively) features prior to melting/crystallisation at $c a .900 \mathrm{~K}$. The DTA signal is not quantitative, i.e., the signal depends on sample and reference masses as well as sample composition. Small unknown impurity peaks for the $\mathrm{RbAg}_{3} \mathrm{Se}_{2}$ trace are indicated with a "*"symbol. b) Solid transition onset temperatures from DTA on heating and cooling for $A_{x} \mathrm{~K}_{1-x} \mathrm{Ag}_{3} \mathrm{Se}_{2}(A=\mathrm{Na}$ or $\mathrm{Rb})$. DTA traces for all mixed-alkali compounds can be located in Figure S12 in the SI. 